\title{
Gender Differences in Alcohol Consumption and Alcohol Related Outcomes: An Empirical Review
}

\author{
Koinis Aristotelis ${ }^{1}$, Mousouros Alexandros ${ }^{2}$, Mermigkas Konstantinos ${ }^{3}$ \\ ${ }^{1}$ Faculty of Human Movement and Quality of Life, Department of Nursing, Sparta, Greece/ Psychiatric Department, General Hospital of \\ Argolida, University of Peloponnese, Argos, Greece \\ ${ }^{2}$ Psychiatric Department, General Hospital of Argolida, Argos, Greece \\ ${ }^{3}$ Faculty of Human Movement and Quality of Life, Department of Nursing, University of Peloponnese, Sparta, Greece
}

\section{Email address:}

telis_psyc@hotmail.com (K. Aristotelis), alexmoussouros@hotmail.com (M. Alexandros), mermigkask@gmail.com (M. Konstantinos)

\section{To cite this article:}

Koinis Aristotelis, Mousouros Alexandros, Mermigkas Konstantinos. Gender Differences in Alcohol Consumption and Alcohol Related Outcomes: An Empirical Review. Journal of Family Medicine and Health Care. Vol. 1, No. 1, 2015, pp. 4-7.

doi: $10.11648 / \mathrm{j} . j \mathrm{fmhc} .20150101 .12$

\begin{abstract}
The present empirical review focuses on the gender differences of alcohol consumption and the course of alcohol related outcomes. As epidemiological findings indicate alcohol use and abuse is much higher in males than females. However, there is a "paradox", women start consuming alcohol much later in life and develop faster adverse alcohol related outcomes than men. Several research findings support this tendency for general pathology and also for brain structure alterations. The purpose of the present study is to give an insight about the course and the outcome of alcohol abuse between genders, by presenting empirical research findings of earlier research (1980s-1990s), and more up to date research (2000s), with the main focus the brain morphological alterations.
\end{abstract}

Keywords: Alcohol, Gender Differences, Adverse Outcomes

\section{Introduction}

The last decades of alcohol research have indicated that several factors associated with excessive alcohol consumption may lead to short-term and long-term adverse outcomes. For instance, there are biological risk factors, such as the genetic risk and the gender differences in the physiological effects of alcohol; and psychosocial risk factors, such as the social sanctions and gender roles, and the drinking motives and expectancies [1]. Several authors have emphasized on gender differences with regards to alcohol consumption and alcohol related outcomes. Epidemiological findings have indicated a great gender difference in alcohol consumption through lifetime. The National Comorbidity Survey (NCS), which used a sample of 8,000 participants below the age of 55 proposed that $12.5 \%$ of males are going to meet the criteria for lifetime alcohol abuse, and $20.1 \%$ of males for lifetime alcohol dependence. On the other hand, the percentages for females of the same sample were much lower, with $6.4 \%$ of women meeting the criteria for lifetime alcohol abuse, and $8.2 \%$ for lifetime alcohol dependence [2]. Additional evidence for alcohol dependence between genders comes by the National Longitudinal Alcohol Epidemiologic
Survey (NLAES). The NLAES with a sample of 42,862 participants over the age of 18 found that $18.6 \%$ of men and $8.4 \%$ of women are going to meet the criteria for lifetime alcohol dependence [3].

The gender difference is not limited only to alcohol consumption but also to the velocity of the adverse outcomes [4]. For heavy drinking women, the development of "liver cirrhosis, alcohol-induced cardiomyopathy, brain atrophy, and cognitive deficits", require less years of alcohol abuse comparably to male heavy drinkers [4], [5]. Additional evidence indicates gender differences in chronic alcohol consumption and the development of the central nervous system malfunction; however, there is no clear explanation of how gender affects the development of central nervous system deficits [4]. The purpose of the present study is to give insight regarding the effects of alcohol on human brain, and the role of gender in the course and the outcome of those brain changes.

Research in the field has indicated specific brain areas affected by excessive alcohol consumption such as, "the neocortex (especially the frontal lobes), the limbic system, 
and the cerebellum" [6]. As aforementioned, epidemiological findings indicate that women are less likely to develop alcohol dependence, and for those who develop problematic drinking the age of onset is much later in life [7]. For females with problematic drinking there is a telescoping effect, which refers to the later alcohol dependence in women [8]. In addition, women have a faster progression to adverse alcohol related outcomes [8].

\section{Previous Research}

Evidence supporting the telescoping effect and the more negative consequences of alcohol in women comparably to men, can be identified in earlier research findings. The study by Jacobson in 1986 indicated "a greater degree of ventricular enlargement, sulsal, interhemispheric and Sylvian fissure widening" in a sample of 26 alcoholic women compared with 41 non-alcoholic women after a CT brain scan [9]. In the same sample, "widening of the cerebellar sulci" was identified only in the alcoholic women [9]. The same author compared the drinking patterns of the alcoholic women sample with a sample of alcoholic males, and reached the conclusion that the female alcoholics had a shorter drinking history compared to alcoholic males. Finally, he concluded that it was not only the shorter duration of the alcohol consumption for alcoholic women, but also the consumption of smaller amounts of alcohol by alcoholic women to reach the same level of brain damage with alcoholic men [9]. Similar findings reported by Mann and his team in 1992, by using CT brain scan [10] These authors compared a sample of alcoholic men with a sample of alcoholic women, and reached the conclusion that the indicated brain anomalies for women (in the same areas as by the study of Jacobson in 1986) resulted by a shorter duration of alcohol consumption in comparison to male alcoholics [10].

\section{Current Trends}

More up to date research comes up with mixed evidence with regards the telescoping effect and the more rapid progression of the adverse alcohol related outcomes [7]. For instance, the study by Hommer and his team in 2001, with a sample of 43 alcoholic men compared with 20 non-alcoholic men, and 36 alcoholic women compared with 19 nonalcoholic women, confirms the telescoping effect and the faster negative alcohol related outcomes for women, after MRI examinations [4]. In specific, they found a decreased "grey and white matter volumes" in the sample of alcoholic men in comparison with the sample of non-alcoholic males. The comparison of the alcoholic women sample with the non-alcoholic women sample, indicated a decreased "grey and white matter volumes", and an increased "CSF volume". It is important to note that the differences in the brain volumes between alcoholic and non-alcoholic women were larger than the differences between alcoholic and nonalcoholic men, especially with regards the grey matter volume [4].

Another up to date study in favor of the telescoping effect and the faster progression of negative alcohol related outcomes for women, is the study by Mann and his team in 2005 [8]. In this study they used a sample of 34 alcoholic men compared with 48 non-alcoholic men, and 42 alcoholic women compared with 34 non-alcoholic women. They found an increase in "sulcal CSF volumes", a decrease in the "brain volume", and "a lower atrophy index (ratio of brain volume over intracranial volume)" for both genders of alcoholics in comparison to non-alcoholics, examined by a CT scan. However, when the authors adjusted the weight differences between alcoholic men and alcoholic women there was no significant difference in the brain alterations. In their study, the more rapid progression of adverse alcohol related outcomes for women was significant [8].

On the other hand, the study by Pfefferbaum and his team in 2001, with a sample of 44 alcoholic men compared with 48 non-alcoholic men, and 42 alcoholic women compared with 37 non-alcoholic women, rejects the telescoping effect and the rapid progression of the negative alcohol related consequences for women, after MRI examinations [7]. The authors reported decreases in "grey and white matter volumes" and increases in "sulci, lateral and third ventricles", for the alcoholic men compared with the non-alcoholic men of their sample. However, this was not the case for women. There were no differences for alcoholic women of their sample in comparison with the non-alcoholic women in any cortical brain structures [7].

Finally, a really interesting finding by Mann and his team in 2005 was the reversibility of the affected brain structures of alcoholic women in comparison with alcoholic men [8]. They found a more significant relationship in alcoholic women after alcohol abstinence between week 1 and week 6 comparably to alcoholic men. In specific, their alcoholic sample showed an increase in "the brain volume and the global atrophy index", and a decrease "in CSF volumes" [8]. However, it is still not clear how this reversibility effect in favor of alcoholic women took place, and more research is needed with regards to the effects of alcohol abstinence between genders.

\section{Conclusions and Future Directions}

As aforementioned, the role of gender with regards the differences in alcohol consumption and the progression of alcohol related adverse outcomes is not clear. It is important to refer to different factors, which may lead to gender differences. When women exposed to the same amount of alcohol intake as men, they show a higher amount of bloodalcohol concentration [1]. Some possible reasons for this imbalance rely on human biology. Usually women have smaller body mass than men. In addition, the amount of alcohol concentration in women's body is higher because women have less amount of water in their body compared with men of the same body mass [1], [8]. Another possible reason may be because of the diminished activity of the 
enzyme "gastric dehydrogenase" in women comparably to men, which leads to the higher amounts of alcohol through the stomach and into the blood stream [1]. Gender differences may also rely on hormonal functions (estrogen for women); however, there are not clear research findings for the last claim [1], [8], [7]. Another possible reason is the lower amount of blood volume in women, by resulting to an increased concentration of alcohol in the tissues. "This is enhanced by a higher ratio of fat to lean tissue, since alcohol is relatively insoluble in the former" [11]. Finally, we should mention that non-alcoholic men have a larger head size and brain volume in comparison with non-alcoholic women. However, it has not been clarified yet if there is gender variability in specific brain structures between healthy males and females [12], [13], [14], [15].

In conclusion, the study of gender differences in alcohol consumption and adverse alcohol related outcomes (brain structures alterations), has started taking place in the 1980s. As epidemiological findings indicate (e.g. NCS, NLAES), lifetime alcohol abuse and lifetime alcohol dependence for males is almost double comparably to females. Moreover, there is supporting evidence in favor of the telescoping effect and the faster adverse alcohol related outcomes for women, not limited only to the brain structure alterations (e.g. "liver cirrhosis, alcohol-induced cardiomyopathy, and brain atrophy") [4], [5]. Even though up to date research mostly supports the telescoping effect, several factors should be considered with caution when we make gender comparisons in relevance to the effects of alcohol. For instance, empirical findings have not yet given a clear explanation if the head size and brain size differences pose an effect on the faster progression of alcohol related outcomes in women [12], [13], [14], [15]. In addition, it is not clear if there is a specific brain structure association with the brain volume difference [12], [13], [14], [15]. The role of hormones (e.g. estrogen) for women has not been clarified with regards the alcohol consumption and the adverse effects of alcohol for women [1], [8], [7]. Based on the aforementioned assumptions, one suggestion may be to conduct a thorough research on the biological aspects of each gender separately in relation to the effects of alcohol, by aiming to have an insight of the underlying mechanisms. Moreover, relevant research on alcohol effects between genders has used only cross sectional designs with relatively small samples. It would have been scientifically interesting to conduct a longitudinal design with a large sample, or ideally a cross-sequential design with a large sample, in order to have a clearer picture of probable causal relations. It is also important to have sample recruitment not only by clinics and groups like the alcoholic anonymous, but also to include people by general practitioners, and people that may not have seeked treatment. This can be conducted by internet ads and newspaper ads in order to have a larger and a more diverse sample. Finally, it is important for each gender comparison to have a body mass adjustment by aiming to eliminate probable bias.

\section{References}

[1] S. Nollen-Hoeksema, and L. Hilt, "Possible contributors to the gender differences in alcohol use and problems," The Journal of General Psychology, vol. 133, pp. 357-374, October 2006.

[2] R. C. Kesler, "The national comorbidity survey (NCS) and its extensions," In Textbook of Psychiatric Epidemiology, M. T. Tsuang, M. Tohen, and P. B. Jones, Eds., Oxford, UK: WilleyBlackwell Publishers Ltd, 2011, pp. 221-237.

[3] B. F. Grant, and D. A. Dawson, "Age at onset of alcohol use and its association with DSM-IV alcohol abuse and dependence: results from the national longitudinal alcohol epidemiologic survey," Journal of Substance Abuse, vol. 9, pp. 103-110, 1997.

[4] D. W. Hommer, R. Momenan, E. Kaizer, and R. R. Rawlings, "Evidence for a gender-related effect of alcoholism on brain volumes," American Journal of Psychiatry, vol. 158, pp. 198204, February 2001.

[5] A. Diehl, B. Croissant, A. Batra, G. Mundle, H. Nakovics, and K. Mann, "Alcoholism in women: is it different in onset and outcome compared to men?" European Archives of Psychiatry and Clinical Neuroscience, 257, vol. 6, pp. 344-351, July 2007.

[6] M. Oskar-Berman, and K. Marinkovic, "Alcohol: effects on neurobehavioral functions and the brain," Neuropsychology Review, vol. 17, pp. 239-257, September 2007.

[7] A. Pfefferbaum, M. Rosenbloom, A. Deskhmuck, and E. V. Sullivan, "Sex differences in the effects of alcohol on brain structure," American Journal of Psychiatry, vol. 158, pp. 188197, February 2001.

[8] K. Mann, K. Ackerman, B. Croissant, G. Mundle, H. Nakovics, and A. Diehl, "Neuroimaging of gender differences in alcohol dependence: are women more vulnerable?" Alcoholism Clinical and Experimental Research, vol. 29, pp. 896-901, May 2005.

[9] R. Jacobson, "Female alcoholics: a controlled CT brain scan and clinical study," British Journal of Addiction, vol. 81, pp. 661-669, May 1986.

[10] K. Mann, A. Batra, A. Gunther, and G. Schroth, "Do women develop alcoholic brain damage more readily than men?" Alcohol Clip Exp Res, vol. 16, pp. 1052-1056, December 1992.

[11] F. Dunne, "Are women more easily damaged by alcohol than men?" British Journal of Addiction, vol. 83, pp.1135-1136, October 1988.

[12] M. De Lacoste, T. Adesanya, and D. Woodward, "Measures of gender differences in the human brain and their relationship to brain weight," Biol Psychiatry, vol. 28, pp. 931-942 December 1990.

[13] S. Breedlove, "Sexual dimorphism in the vertebrate nervous system," Journal of Neuroscience, vol. 12, pp. 4133-4142, November 1992.

[14] S. Clarke, R. Kraftsik, H. van der Loos, and G. Innocenti, "Forms and measures of adult and developing human corpus callosum: is there sexual dimorphism?" Journal of Comparative Neurology, vol. 280, pp. 213-230, February 1989. 
[15] L. S. Allen, M. F. Richey, Y. M. Chai, and R. A. Gorski, "Sex differences in the corpus callosum of the living human being," The Journal of Neuroscience, vol. 11, pp. 933-942, April 1991. 